ORIGINAL RESEARCH

\title{
Protection Against Cold in Prehospital Care: Wet Clothing Removal or Addition of a Vapor Barrier
}

\author{
Otto Henriksson, MD; Peter J. Lundgren, MD; Kalev Kuklane, PhD; Ingvar Holmér, PhD; \\ Gordon G. Giesbrecht, PhD; Peter Naredi, MD PhD; Ulf Bjornstig, MD PhD
}

From the Division of Surgery, Department of Surgical and Perioperative Sciences, Umea University, Umeå, Sweden (Drs Henriksson, Lundgren, Naredi, and Bjornstig); the Thermal Environment Laboratory, Department of Design Sciences, Faculty of Engineering, Lund University, Lund, Sweden (Drs Kuklane and Holmér); and the Laboratory for Exercise and Environmental Medicine, University of Manitoba, Winnipeg, Manitoba, Canada (Dr Giesbrecht).

\begin{abstract}
Objective.-The purpose of this study was to evaluate the effect of wet clothing removal or the addition of a vapor barrier in shivering subjects exposed to a cold environment with only limited insulation available.

Methods.-Volunteer subjects $(n=8)$ wearing wet clothing were positioned on a spineboard in a climatic chamber $\left(-18.5^{\circ} \mathrm{C}\right)$ and subjected to an initial 20 minutes of cooling followed by 30 minutes of 4 different insulation interventions in a crossover design: 1) 1 woolen blanket; 2) vapor barrier plus 1 woolen blanket; 3) wet clothing removal plus 1 woolen blanket; or 4) 2 woolen blankets. Metabolic rate, core body temperature, skin temperature, and heart rate were continuously monitored, and cold discomfort was evaluated at 5-minute intervals.

Results.-Wet clothing removal or the addition of a vapor barrier significantly reduced metabolic rate (mean difference $\left.\pm \mathrm{SE} ; 14 \pm 4.7 \mathrm{~W} / \mathrm{m}^{2}\right)$ and increased skin temperature rewarming $\left(1.0^{\circ} \pm\right.$ $0.2^{\circ} \mathrm{C}$ ). Increasing the insulation rendered a similar effect. There were, however, no significant differences in core body temperature or heart rate among any of the conditions. Cold discomfort (median; interquartile range) was significantly lower with the addition of a vapor barrier $(4 ; 2-4.75)$ and with 2 woolen blankets $(3.5 ; 1.5-4)$ compared with 1 woolen blanket alone $(5 ; 3.25-6)$.

Conclusions.- In protracted rescue scenarios in cold environments with only limited insulation available, wet clothing removal or the use of a vapor barrier is advocated to limit the need for shivering thermogenesis and improve the patient's condition on admission to the emergency department.
\end{abstract}

Key words: hypothermia, heat loss, thermal insulation, emergency medical services

\section{Introduction}

Admission hypothermia, defined as a core body temperature less than $35^{\circ} \mathrm{C}$, is an independent predictor of increased mortality and morbidity in trauma patients. ${ }^{1-6}$ The reported incidence of hypothermia varies from $1.6 \%$ to $43 \%$ with severity and mechanism of injury, the presence of shock, duration of evacuation, and prehospital induction of anesthesia as predictive variables of the trauma patient arriving hypothermic to the emergency department. ${ }^{7-10}$

In addition to immediate care for life-threatening conditions, early application of adequate insulation to reduce heat loss and prevent body core cooling is an

Corresponding author: Otto Henriksson, MD, Division of Surgery, Department of Surgical and Perioperative Sciences, Umeå University, SE-90185 Umeå, Sweden (e-mail: otto.henriksson@ surgery.umu.se). important part of prehospital trauma care. ${ }^{1-13}$ If the patient is wet, most prehospital guidelines on protection against cold recommend the removal of wet clothing before insulation or the use of a vapor barrier between the wet patient and the insulation. ${ }^{14-20}$ In the field, however, the removal of wet clothing might be impeded because of harsh environmental conditions or the patient's condition and injuries. Also, encapsulation in a vapor barrier might restrict necessary access and monitoring of the patient during transport.

Using a thermal manikin, we previously demonstrated that independent of insulation thickness $(1,2$, or 7 woolen blankets), wet clothing removal or the addition of a vapor barrier reduced total heat loss by about onefourth $(19 \%-31 \%)$ in a cold environment $\left(-15.4^{\circ} \mathrm{C}\right)$ and one-third $(27 \%-42 \%)$ in a warm environment $\left(+11.0^{\circ} \mathrm{C}\right)$; the absolute reduction, however, was greater 
in the cold environment and with fewer blankets applied. ${ }^{21} \mathrm{~A}$ similar reduction in total heat loss was also achieved by increasing the insulation from 1 to 2 blankets or from 2 to 7 blankets. These findings suggest that the clinical relevance of wet clothing removal or the addition of a vapor barrier is greater in a sustained cold environment with only limited insulation available.

To verify these findings and evaluate the effect of wet heat loss reduction on human thermoregulation, a follow-up human trial was designed. The objective of this study was to evaluate the effect of wet clothing removal or the addition of a vapor barrier in shivering subjects exposed to a cold environment with only limited insulation available. The primary outcome measure was metabolic rate, with core body temperature, skin temperature, heart rate, and cold discomfort as secondary outcome variables. Our hypothesis was that wet clothing removal or the addition of a vapor barrier would significantly reduce shivering thermogenesis and abate peripheral vasoconstriction, thus lowering the metabolic rate, increasing skin temperature, and improving cold discomfort. We anticipated no significant differences in core body temperature.

\section{Methods}

\section{DESIGN, SETTING, AND SUBJECTS}

The study was conducted in October 2010 at the Thermal Environment Laboratory, Lund University, Lund, Sweden. Ethical approval was obtained from the Regional Ethical Review Board in Umea.

The study was designed to evaluate shivering thermogenesis, core body temperature, skin temperature, heart rate, and cold discomfort in cold-stressed subjects wearing wet clothing during an initial cold exposure followed by 4 different insulation interventions: 1) 1 woolen blanket (control condition); 2) vapor barrier plus 1 woolen blanket; 3) wet clothing removed plus 1 woolen blanket; or 4) 2 woolen blankets.

Four male and 4 female nonsmoking healthy students at the Faculty of Medicine, Lund University, volunteered for participation (Table 1). Each subject served as his or her own control and participated in all 4 conditions. Each subject was randomly assigned to complete the trials in a unique order following a balanced design, scheduled at about the same time of day on 4 separate occasions, 1 week apart. The subjects abstained from smoking, alcohol, or drug use, had a minimum of 6 hours of sleep and adequate meals, and avoided physical exertion during the 24 hours before the trials.

The climatic chamber $(2.4 \times 2.4 \times 2.4 \mathrm{~m})$ was set to $-20^{\circ} \mathrm{C}$, and in concordance with the previous study using a thermal manikin, ${ }^{21} 1$ and 2 woolen blankets (Swedish Rescue Agency) were chosen to provide low $(2.8 \pm 0.2$ clo) and moderate $(3.8 \pm 0.1$ clo) insulation in relation to the ambient temperature $\left(1 \mathrm{clo}=0.155 \mathrm{~m}^{2 \circ} \mathrm{C} / \mathrm{W}\right)$. Each blanket measured approximately $199 \times 150 \times 0.5$ $\mathrm{cm}$ and weighed $2.150 \mathrm{~g}$, ie, $2.15 \mathrm{~kg}$. The vapor barrier was made up of 2 large plastic bags taped together to form a large sack measuring approximately $250 \times 85 \mathrm{~cm}$ with a weight of $250 \mathrm{~g}$. The wet clothing worn by the subjects consisted of light, 2-piece cotton/polyester thermal underwear (Swedish Armed Forces) with a total dry weight of approximately $575 \mathrm{~g}$.

\section{MONITORING}

Ambient air temperature was continuously monitored using 3 sensors (PT 100, Pico Technology Ltd., UK; $\pm 0.03^{\circ} \mathrm{C}$ ) positioned in level with the supine subject, adjacent to the ankles, the mid trunk, and the head. No wind was applied, but intrinsic airflow in the climatic chamber was checked with a directionally independent

Table 1. Characteristics of subjects

\begin{tabular}{llcccccc}
\hline Subject & Sex & Age $(y)$ & Height $(\mathrm{cm})$ & Weight $(\mathrm{kg})$ & BMI $\left(\mathrm{kg} / \mathrm{m}^{2}\right)^{a}$ & ${\text { BSA }\left(\mathrm{m}^{2}\right)^{b}}$ & ${\text { Body fat }(\%)^{c}}^{c}$ \\
\hline 1 & $\mathrm{M}$ & 32 & 182 & 81 & 24 & 2.0 & 13 \\
2 & $\mathrm{M}$ & 25 & 186 & 87 & 25 & 2.1 & 18 \\
3 & $\mathrm{M}$ & 24 & 196 & 89 & 23 & 2.2 & 12 \\
4 & $\mathrm{M}$ & 24 & 185 & 82 & 24 & 2.1 & 18 \\
5 & $\mathrm{~F}$ & 25 & 172 & 61 & 21 & 1.7 & 21 \\
6 & $\mathrm{~F}$ & 21 & 166 & 56 & 20 & 1.6 & 14 \\
7 & $\mathrm{~F}$ & 21 & 162 & 54 & 21 & 1.6 & 18 \\
8 & $\mathrm{~F}$ & 23 & 169 & 62 & 22 & 1.7 & 16 \\
\hline
\end{tabular}

\footnotetext{
${ }^{a}$ Calculated according to McArdle et al. ${ }^{22}$

${ }^{b}$ Calculated according to Du Bois and Du Bois. ${ }^{23}$

${ }^{c}$ Calculated according to Durnin and Womersley. ${ }^{24}$

BMI, body mass index; BSA, body surface area.
} 
wind speed sensor (SWA01, Swema AB, Sweden; $\pm 0.02 \mathrm{~m} / \mathrm{s}$ ).

Shivering thermogenesis was evaluated by continuous monitoring of respiratory exchange ratio (RER) and oxygen consumption $\left(\dot{\mathrm{V}}_{2}\right)$ using a tight-fitting opencircuit breathing mask with an insulated sampling hose (Metamax I, Cortex Biophysik GmbH, Leipzig, Germany). Metabolic rate (M) in watts per meter squared $\left(A_{D}=\right.$ body surface area, given by the Du Bois formula $^{23}$ ) was calculated according to the partial method: $\mathrm{M}=5.873(0.23 \mathrm{RER}+0.77) \dot{\mathrm{V}}_{2} / \mathrm{A}_{\mathrm{D}}{ }^{25}$

Core body temperature was evaluated by continuous monitoring of esophageal temperature. The close proximity of the esophagus to the descending aorta and the left auricle provides an accurate and highly sensitive measurement of arterial blood temperature and thus core body temperature. ${ }^{26-28}$ A flexible temperature probe (ER400-9, Smiths Medical, Ltd, Ashford, UK, $\pm 0.2^{\circ} \mathrm{C}$ ) was gently inserted through one of the nostrils down to a retrocardiac position in the esophagus, the individual insertion depth determined from the subject's body height, according to Mekjavic et al. ${ }^{29}$

Skin temperature was continuously measured using thermal transducers (ACC-001, Rhopoint Components Ltd, East Grinstead, UK, $\pm 0.2^{\circ} \mathrm{C}$ ) taped to the skin at 8 predefined locations (forehead, upper arm, forearm, hand, chest, scapula, calf, and thigh), enabling areaweighted calculations of mean skin temperature. ${ }^{28}$

Heart rate was continuously monitored using a pulse belt (Sport Tester, Polar Electro, Oy, Kempele, Finland) with transmission of the electrocardiographic signal to the Metamax equipment.

Cold discomfort was evaluated using a numerical rating scale, on which the subjects assessed the thermal state of their whole body, not specific body parts, providing values from 0 to 10 , where 0 indicated not being cold at all and 10 indicated unbearably cold. ${ }^{30}$ Cold discomfort ratings were sampled at 5-minute intervals. All other data were continuously recorded at 10 -second intervals.

\section{PROTOCOL}

Before and between the trials, subject clothing and insulation materials were kept dry and at room temperature. The subject first dressed in standardized underwear consisting of short fleece underpants and for women also a fleece bra. Skin temperature transducers and pulse belt were applied. The esophageal probe was inserted, and the breathing mask was positioned and checked for leaks before connecting to the sampling hose. Wearing a bathrobe, 2 pairs of dry woolen socks, and a fleece cap, the subject then sat quietly on a chair in room temperature for 15 minutes of baseline data collection. Meanwhile the thermal underwear was wetted according to a standardized rinsing program using an industrial washing machine. The water content of the wetted clothing was measured using a precision scale (K240, Mettler Toledo Inc, Greifensee, Switzerland, $\pm 5 \mathrm{~g}$ ). After baseline completion the subject immediately dressed in the wet thermal underwear. To protect from local cold injuries, high insulation mittens and boot covers were put on.

The subject then entered the cold chamber and lay down on an ordinary spineboard (Baxstrap, Laerdal Medical AS, Stavanger, Norway) with an additional sleeping mattress on top underneath the participant. One of the medical doctors responsible for the study (O.H. or P.L.) accompanied the subject in the cold chamber during the whole trial, collecting cold discomfort ratings, applying the subsequent insulation, and assessing the medical condition of the subject. After 20 minutes of cold exposure the insulation was applied. If assigned, wet clothing was cut off and carefully removed or the vapor barrier sack was pulled over the subject from the feet up and tightly folded around the neck. Finally, the assigned number of woolen blankets were laid on top of the subject from the neck down and tightly folded in under the spineboard. After 30 minutes of insulation intervention, the trial was completed and the subject exited the cold chamber. Wet clothing was taken off, monitoring equipment was removed, and the subject then had a warm shower until restoration of thermal comfort.

\section{DATA ANALYSIS}

Metabolic rate, heart rate, and cold discomfort were analyzed as mean values during steady-state conditions at baseline $\left(\mathrm{t}_{-10-0}\right)$, cooling $\left(\mathrm{t}_{10-20}\right)$, and intervention $\left(\mathrm{t}_{30-50}\right)$. Core body and mean skin temperatures were analyzed at the end of baseline $\left(\mathrm{t}_{0}\right)$ and cooling $\left(\mathrm{t}_{20}\right)$ and as a change from the start to the end of the intervention $\left(t_{20-50}\right)$. Normally distributed continuous data were compared using a repeated measures analysis of variance. When a significant main effect was revealed, Student's $t$ test for pairwise comparisons with Fisher's least significant difference adjustment for multiple comparisons was performed to identify differences among the 4 conditions. Ordinal data were compared using Friedman's test. When a significant main effect was revealed, the Wilcoxon signed ranks test was performed to identify differences among the 4 conditions. Cold discomfort ratings from one of the trials were lost during the data processing and for this parameter cases were excluded testby-test during the analysis. Statistical significance was defined as a probability value of less than .05 (2-sided), and SPSS 18.0 software (SPSS, Inc, Chicago, IL) was used for the analysis. 


\section{Results}

\section{ENVIRONMENT}

Air temperature for all conditions was $-18.5^{\circ} \pm 0.2^{\circ} \mathrm{C}$ (mean $\pm \mathrm{SD}$ ), and intrinsic wind speed in the climatic chamber was $0.22 \pm 0.07 \mathrm{~m} / \mathrm{s}$. Water content of the wet clothing before cold exposure was $1232 \pm 91 \mathrm{~g}$ for all trials, with no significant differences among conditions.

\section{METABOLIC RATE}

Mean metabolic rate during baseline $\left(66 \pm 12 \mathrm{~W} / \mathrm{m}^{2}\right)$ increased 1.6-fold during initial cold exposure (107 \pm 16 $\mathrm{W} / \mathrm{m}^{2}$ ) with no significant differences among conditions. During the insulation interventions, mean metabolic rate decreased in all conditions but was significantly lower with wet clothing removal $\left(85 \pm 11 \mathrm{~W} / \mathrm{m}^{2} ; P=.036\right)$, the addition of a vapor barrier $\left(84 \pm 17 \mathrm{~W} / \mathrm{m}^{2} ; P=\right.$ $.022)$, or when 2 woolen blankets were applied $(81 \pm 10$ $\left.\mathrm{W} / \mathrm{m}^{2} ; P=.008\right)$ compared with 1 woolen blanket alone $\left(98 \pm 12 \mathrm{~W} / \mathrm{m}^{2}\right.$; Figure 1, Table 2).

\section{CORE BODY TEMPERATURE}

There were no significant differences among conditions in core body temperature at baseline $\left(36.8^{\circ} \pm 0.3^{\circ} \mathrm{C}\right)$ or at the end of initial cold exposure $\left(36.9^{\circ} \pm 0.5^{\circ} \mathrm{C}\right)$. During the insulation interventions there was a small reduction in core body temperature $\left(-0.3^{\circ} \pm 0.2^{\circ} \mathrm{C}\right)$ with no significant differences among conditions $(P=.631$; Figure 2, Table 2).

\section{SKIN TEMPERATURE}

There were no significant differences among conditions in mean skin temperature at baseline $\left(33.5^{\circ} \pm 0.3^{\circ} \mathrm{C}\right)$ or at the end of the initial cold exposure $\left(24.9^{\circ} \pm 0.9^{\circ} \mathrm{C}\right)$. During the insulation interventions, the mean skin temperature increase was significantly higher with wet clothing removal $\left(2.5^{\circ} \pm 0.7^{\circ} \mathrm{C} ; P=.001\right)$, the addition of a vapor barrier $\left(2.7^{\circ} \pm 0.8^{\circ} \mathrm{C} ; P=.009\right)$, or when 2 woolen blankets were applied $\left(2.3^{\circ} \pm 0.7^{\circ} \mathrm{C} ; P=.003\right)$ compared with 1 woolen blanket alone $\left(1.5^{\circ} \pm 0.4^{\circ} \mathrm{C}\right.$; Figure 3 , Table 2$)$.

\section{HEART RATE}

Mean heart rate remained at about the same level during the initial cold exposure $(81 \pm 8$ beats $/ \mathrm{min})$ as at baseline ( $83 \pm 11$ beats/min) with no significant differences among conditions. During insulation interventions mean heart rate was decreased ( $67 \pm 5$ beats/min) with no significant differences among conditions $(P=.159$; Figure 4, Table 2).

\section{COLD DISCOMFORT}

Median cold discomfort increased from 0 (interquartile range [IQR], 0-0) during baseline to 6.25 (IQR, 4.5-7.75) during initial cold exposure with no significant differences among conditions. During the insulation interventions the median cold discomfort was reduced for all conditions but was significantly lower with the addition of a vapor barrier (4; IQR, 2-4.75; $P=0.008$ ), or when 2 woolen

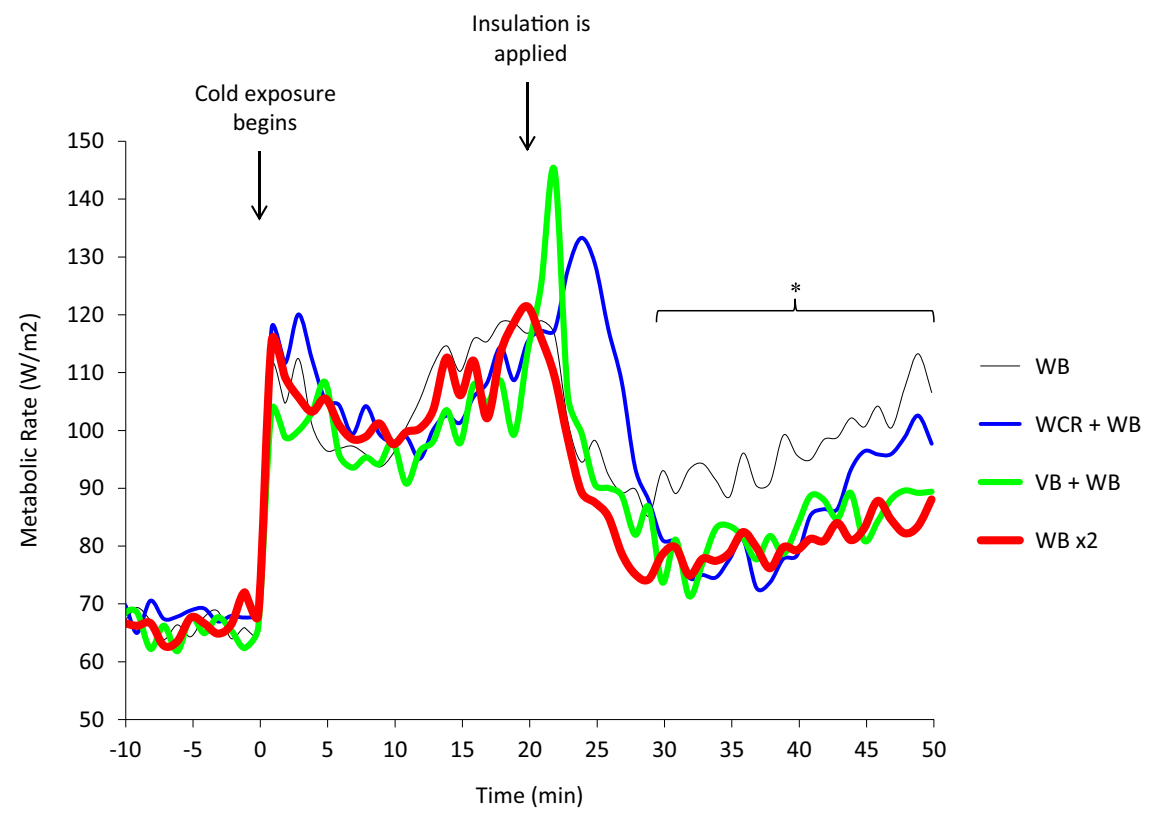

Figure 1. Metabolic rate during 4 insulation protocols in a cold environment (mean, $n=8$ ) was significantly lower during last 20 minutes of insulation with wet clothing removal (WCR) +1 woolen blanket $(\mathrm{WB})$ : blue line; vapor barrier $(\mathrm{VB})+1 \mathrm{WB}$ : green line; and 2 woolen blankets $(\mathrm{WB} \times 2)$ : red line; compared with $1 \mathrm{WB}$ alone: black line $(P<.05)$. 
Table 2. The effect on thermoregulation and cold discomfort ${ }^{a}$

\begin{tabular}{lccccc}
\hline Insulation protocol & $\begin{array}{c}\text { Metabolic rate } \\
\left(\mathrm{W} / \mathrm{m}^{2}\right)\end{array}$ & $\begin{array}{c}\text { ACore body } \\
\text { temperature }\left({ }^{\circ} \mathrm{C}\right)\end{array}$ & $\begin{array}{c}\text { AMean skin } \\
\text { temperature }\left({ }^{\circ} \mathrm{C}\right)\end{array}$ & $\begin{array}{c}\text { Heart rate } \\
(\text { beats/min })\end{array}$ & $\begin{array}{c}\text { Cold discomfort } \\
(0-10)\end{array}$ \\
\hline $\begin{array}{l}\text { One woolen blanket } \\
\text { Wet clothing } \\
\text { removal + one } \\
\quad \text { woolen blanket }\end{array}$ & $98 \pm 12$ & $-0.4 \pm 0.2$ & $1.5 \pm 0.4$ & $69 \pm 6$ & $5(3.25-6)$ \\
$\begin{array}{l}\text { Vapor barrier + one } \\
\quad \text { woolen blanket }\end{array}$ & $84 \pm 17^{b}$ & $-0.3 \pm 0.2$ & $2.5 \pm 0.7^{b}$ & $70 \pm 7$ & $3(2-5)$ \\
$\begin{array}{l}\text { Two woolen } \\
\text { blankets }\end{array}$ & $81 \pm 10^{b}$ & $-0.3 \pm 0.2$ & $2.7 \pm 0.8^{b}$ & $67 \pm 8$ & $4(2-4.75)^{c}$ \\
\hline
\end{tabular}

\footnotetext{
${ }^{a}$ Volunteer subjects $(\mathrm{n}=8)$ wearing wet clothing in a climatic chamber at $-18.5^{\circ} \mathrm{C}$ with still wind conditions. Values are mean $\pm \mathrm{SD}$ or median (interquartile range) during 4 insulation protocols.

${ }^{b}$ Significantly different from 1 woolen blanket (repeated-measures analysis of variance), $P<.05$.

${ }^{c}$ Significantly different from one woolen blanket (Friedman's test), $P<.05$.
}

blankets were applied, (3.5; IQR, 1.5-4; $P=0.031$ ), compared with 1 woolen blanket alone, (5; IQR, 3.25-6; Figure 5, Table 2). There was, however, no statistically significant difference with wet clothing removal (3; IQR, $2-5 ; P=0.094)$ compared with 1 woolen blanket alone.

\section{Discussion}

\section{OVERVIEW}

Simulating a prehospital rescue scenario, this study evaluated the effect on metabolic rate, core body temperature, skin temperature, heart rate, and cold discomfort in 8 healthy cold-stressed subjects wearing wet clothing during an initial 20 minutes of cold exposure in $-18.5^{\circ} \mathrm{C}$ followed by 30 minutes of 4 different insulation interventions: 1) 1 woolen blanket; 2) vapor barrier plus 1 woolen blanket; 3 ) wet clothing removed plus 1 woolen blanket; or 4) 2 woolen blankets.

The results revealed that mean metabolic rate was significantly lower and skin temperature rewarming was significantly higher with wet clothing removal, the addition of a vapor barrier, or when 2 woolen blankets were applied, compared with 1 woolen blanket alone. Cold discomfort was significantly lower with the addition of a vapor barrier or when 2 woolen blankets were applied, compared with 1 woolen blanket alone. There

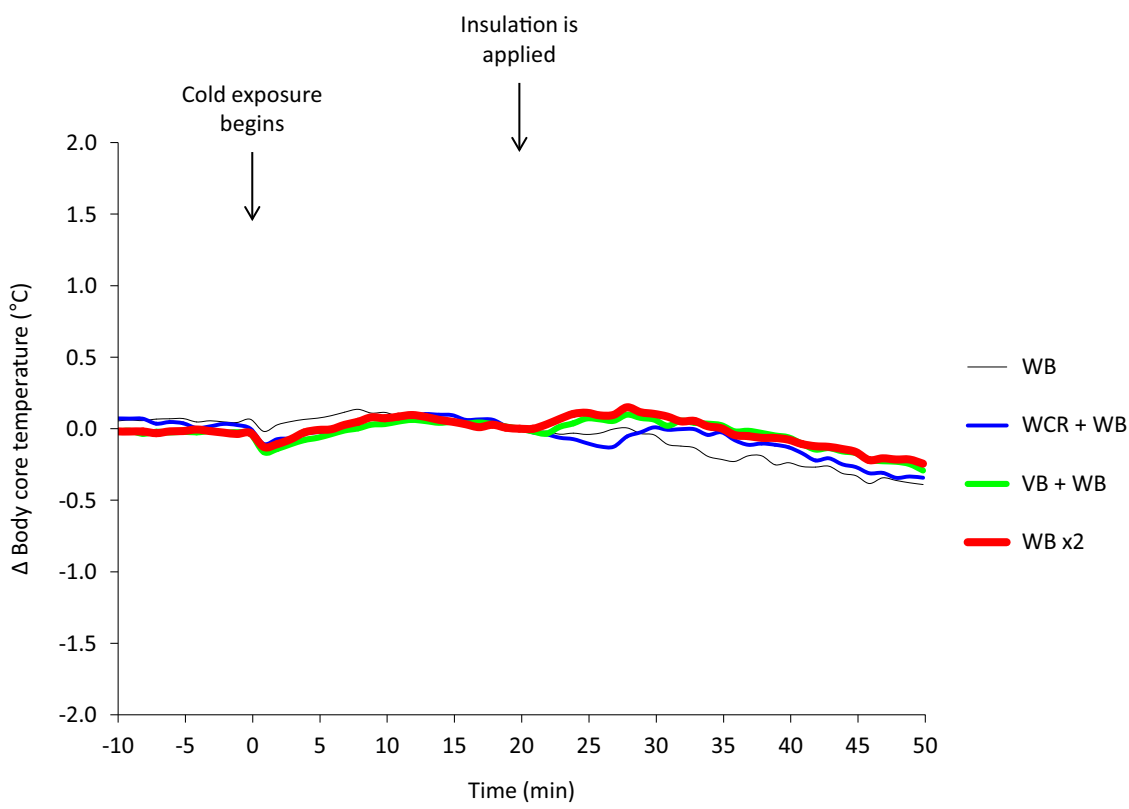

Figure 2. Core body (esophageal) temperature change from insulation applied during 4 insulation protocols in a cold environment (mean, $\mathrm{n}=8$ ). $\mathrm{WB}$, woolen blanket (black line); WCR, wet clothing removal + WB (blue line); VB, vapor barrier + WB (green line); WB $\times 2$, two woolen blankets (red line). 


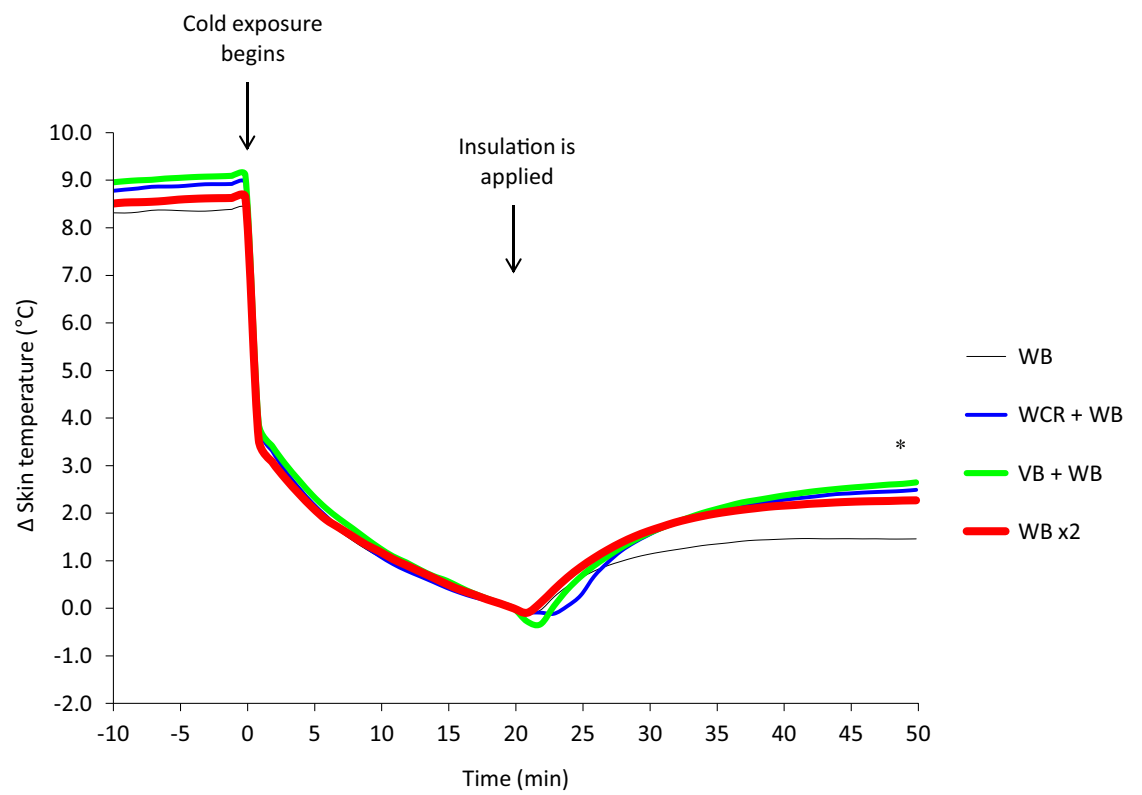

Figure 3. Mean skin temperature change from insulation applied during 4 insulation protocols in a cold environment $($ mean, $n=8)$. There was significantly higher rewarming with wet clothing removal (WCR) + woolen blanket (WB): blue line; vapor barrier (VB) + WB: green line; and 2 woolen blankets $(\mathrm{WB} \times 2)$ : red line; compared with $1 \mathrm{WB}$ alone: black line $(P<.05)$.

were, however, no significant differences among conditions in mean heart rate or core body temperature.

\section{POSSIBLE MECHANISMS FOR RESULTS}

During the initial cold exposure, heat loss from the skin and the subsequent vasoconstriction rendered a significant decline in mean skin temperature. This cold stimulus instantly triggered shivering and a significant increase in metabolic heat production compared with the resting metabolic rate during baseline. The cold-induced vasoconstriction and the increase in endogenous heat production maintained core body temperature at or near normal levels throughout the initial cold exposure.

During the insulation interventions, shivering declined and a slight peripheral rewarming occurred in all

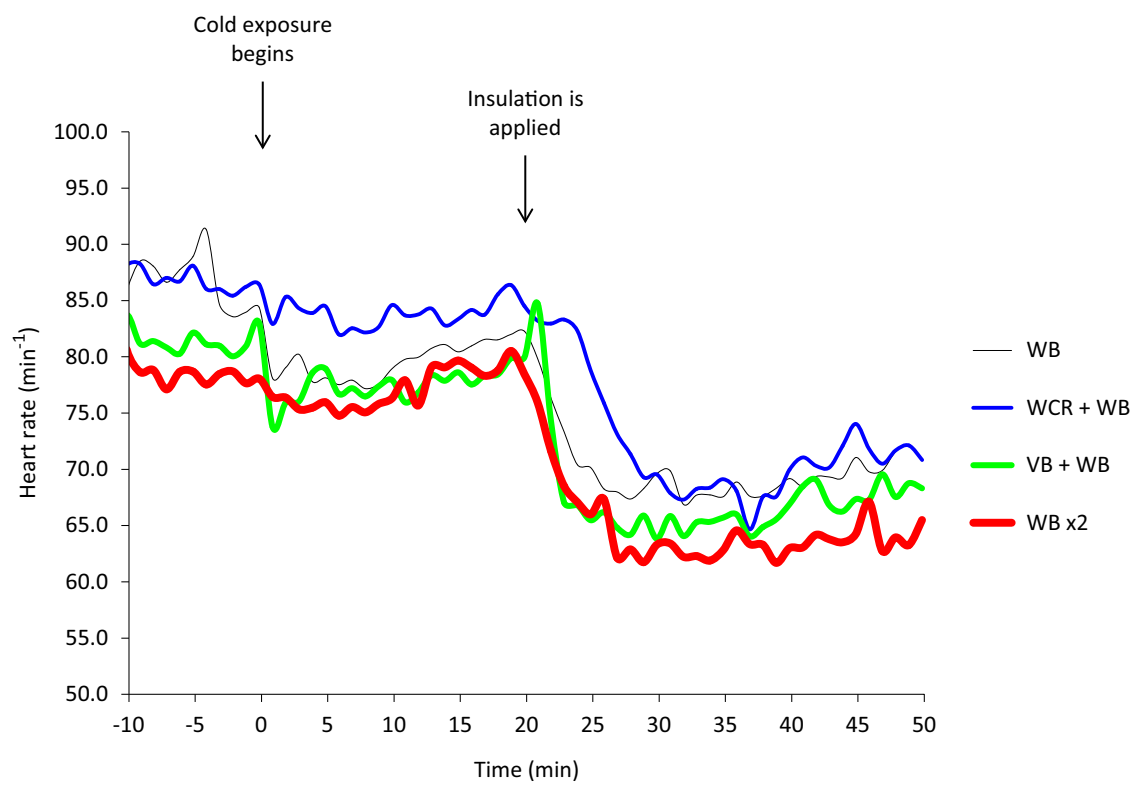

Figure 4. Heart rate during 4 insulation protocols in a cold environment (mean, $\mathrm{n}=8$ ). WB, woolen blanket (black line); WCR, wet clothing removal + WB (blue line); VB, vapor barrier + WB (green line); WB $\times 2$, two woolen blankets (red line). 


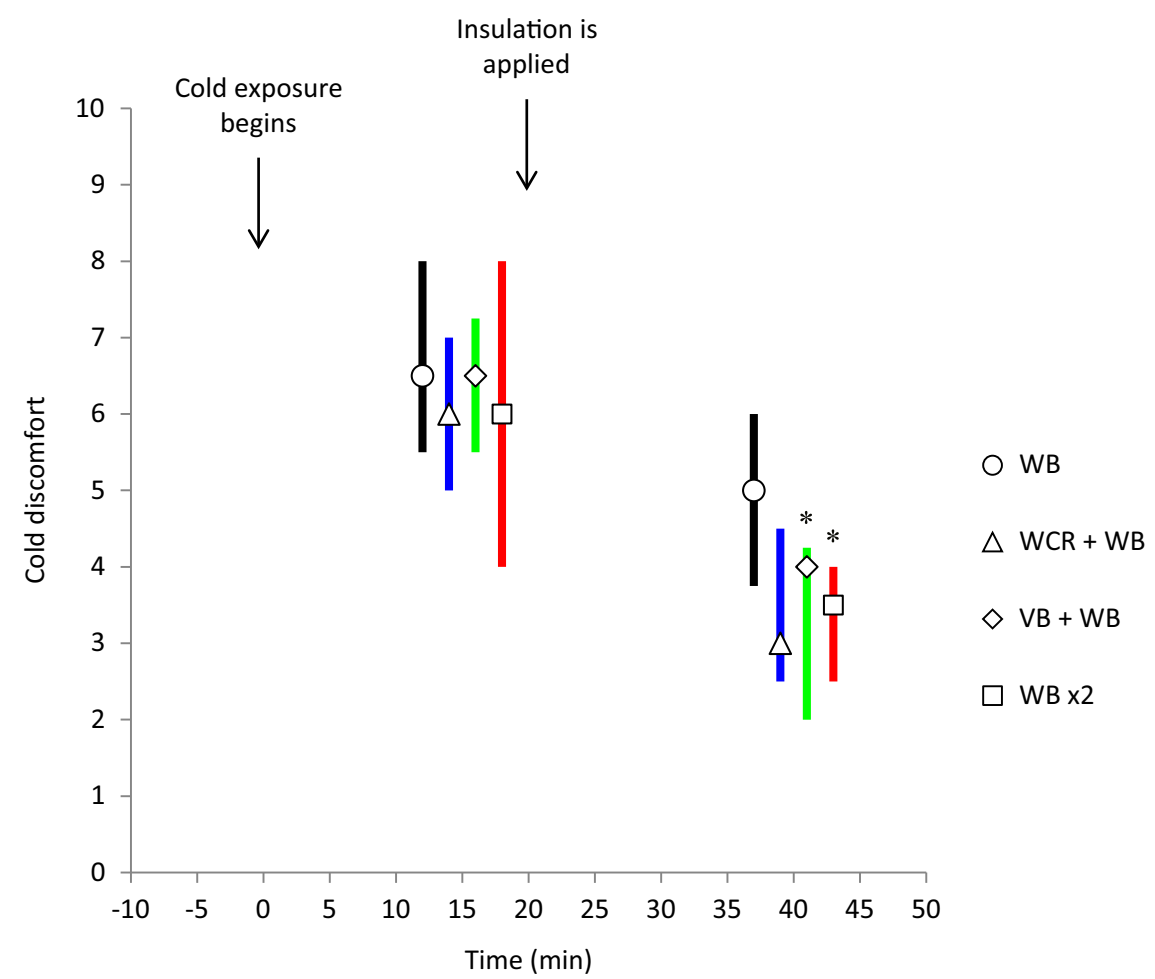

Figure 5. Cold discomfort at initial cooling (10-20 minutes) and with insulation applied (30-50 minutes) during 4 insulation protocols in a cold environment (median; IQR, separated in time for simplicity, $\mathrm{n}=8$ ) was significantly lower during the last 20 minutes of insulation with vapor barrier $(\mathrm{VB})+$ woolen blanket $(\mathrm{WB})$ : green line, and 2 woolen blankets $(\mathrm{WB} \times 2)$ : red line, compared with $1 \mathrm{WB}$ alone: black line $(P<.05)$. The change with wet clothing removal (WCR) + WB: blue line, was not statistically significant.

conditions with mean skin temperature rewarming being significantly higher and metabolic rate being significantly lower with wet clothing removal, the addition of a vapor barrier, or when 2 woolen blankets were applied compared with 1 woolen blanket alone. These findings coincide with the results from our previous thermal manikin study with wet clothing, in which heat loss was significantly reduced with wet clothing removal, the addition of a vapor barrier, or when 2 woolen blankets were applied compared with 1 woolen blanket alone. ${ }^{21}$

Although heat loss was reduced, the continuing cold exposure rendered a small decline in core body temperature during all insulation interventions. The greater heat loss with 1 woolen blanket alone was counteracted by a greater endogenous heat production, and thus core body temperature was not affected by the different insulation interventions.

Shivering thermogenesis requires an increase in oxygen delivery to the muscles, primarily met by an increase in cardiac output. ${ }^{31}$ Although metabolic rate was greater with 1 woolen blanket alone, we found no differences among conditions in mean heart rate during the insulation interventions. As all subjects in this study were young and fit, the greater metabolic rate and thus cardiac output demands with 1 woolen blanket alone were probably met by an increase in stroke volume rather than an increase in heart rate. ${ }^{32}$

Cold discomfort was reduced for all conditions during insulation interventions. However, the differences in metabolic rate and skin temperature rewarming among conditions probably explain why cold discomfort was significantly lower with the addition of a vapor barrier or the application of 2 woolen blankets compared with 1 woolen blanket only. There was also a tendency to a lower cold discomfort with wet clothing removal compared with 1 woolen blanket alone, although this was not statistically significant.

\section{PRACTICAL IMPLICATIONS}

In prehospital trauma care, early application of adequate insulation is of utmost importance to reduce cold stress, limit body core cooling, and prevent deterioration of the patient's condition. ${ }^{11-13}$ If the patient is wet, heat loss will be significantly increased because of evaporation and wet conduction through insulation materials. ${ }^{21}$ Thus, most prehospital guidelines against cold recommend the removal of wet clothing before insulation or the use of a 
vapor barrier between the patient and the insulation. ${ }^{14-20}$ However, in the field, removal of wet clothing might be hazardous, especially with a suspected spinal injury when any unnecessary movements should be avoided, ${ }^{33}$ or if the patient is severely hypothermic, in which case rough handling might provoke spontaneous ventricular fibrillation. ${ }^{11}$ Removal of wet clothing might also be time-consuming and thus contraindicated in the presence of shock and suspected internal bleedings in which case on-scene duration should be limited. ${ }^{33}$ Encapsulation in a vapor barrier might be easier to achieve but bears the potential disadvantage of restricting necessary access and monitoring of the patient during transport.

Few studies have been conducted that evaluate these measures. Thomassen et $\mathrm{al}^{34}$ recently demonstrated that the addition of a plastic sheet under an ordinary ambulance blanket improved skin temperature in subjects wearing wet clothing in a cold $\left(5^{\circ} \mathrm{C}\right)$ and windy $(3 \mathrm{~m} / \mathrm{s})$ environment. There were, however, no significant differences in metabolic rate or thermal sensation. The reason that there were no differences in metabolic rate might be because the ambulance blanket itself provided the required insulation for the ambient conditions selected. This conclusion is supported by the fact that metabolic rate returned to near baseline levels after insulation was applied. In that case, the additional heat loss reduction supplied by the vapor barrier would have a limited effect.

In this study, with only limited insulation available in a more profound cold environment, both wet clothing removal and the addition of a vapor barrier significantly reduced the need for shivering thermogenesis (mean difference $\pm \mathrm{SE} ; 14 \pm 4.7 \mathrm{~W} / \mathrm{m}^{2}$ ) and improved peripheral rewarming (mean difference $\pm \mathrm{SE} ; 1.0^{\circ} \pm$ $0.2^{\circ} \mathrm{C}$ ). Increasing the insulation from 1 to 2 woolen blankets rendered a similar effect. Although the clinical relevance of about $1{ }^{\circ} \mathrm{C}$ difference in mean skin temperature rewarming seems small, a metabolic rate reduction of about $15 \%$ might be of great clinical importance in conjunction with major trauma, preserving oxygen availability for vital organs.

Thus, if the patient can be readily transferred into a warm environment, such as a heated transportation unit, sufficient insulation is easy to achieve, and the need for wet clothing removal or the addition of a vapor barrier is limited. However, in a sustained cold environment with limited insulation available, such as is often the case in protracted evacuations in harsh conditions or in mass casualty situations, the removal of wet clothing or the addition of a vapor barrier might be of great importance to reduce heat loss and limit the need for shivering thermogenesis, thus relieving the patient from unnecessary cardiac and respiratory stress. If endogenous heat production is impaired because of major trauma or circulatory shock, ${ }^{35}$ or because of profound hypothermia, ${ }^{36}$ the removal of wet clothing or the addition of a vapor barrier is likely to be even more important to reduce heat loss and prevent body core cooling.

\section{LIMITATIONS}

This study is unique in its design specifically evaluating the effect on human thermoregulation by wet clothing removal or the addition of a vapor barrier in a cold environment with limited insulation available. Although there were a limited number of participants in this study, the crossover design enabled comparative evaluation of the insulation interventions. However, the results should be interpreted in the case of optimal conditions for the different insulation interventions applied to healthy uninjured subjects. In a real life scenario, the age, injuries, and physical condition of the patient might affect heat loss and thermoregulation capabilities as well as practical aspects of possible insulation interventions. Also, in protracted on-scene durations, moderate or even high wind conditions are likely to be encountered. If wind would have been present, it might have affected the results in favor of the vapor barrier, as it also provides a windproof layer to the insulation ensemble, thus limiting convective heat loss. ${ }^{37}$ The relatively short duration of both cold exposure and the subsequent insulation intervention should also be considered. Specifically there was a tendency for an increase in metabolic rate at the end of the insulation intervention with wet clothing removal. Further studies should be designed to evaluate the combined effect of the vapor barrier as a wind and vapor-resistant layer.

\section{Conclusions}

In protracted rescue scenarios in cold environments with only limited insulation available, wet clothing removal or the addition of a vapor barrier is advocated to reduce heat loss, limit the need for shivering thermogenesis, and improve the patient's condition on admission to the emergency department.

\section{Acknowledgment}

This research was supported by The National Board of Health and Welfare, Sweden.

\section{References}

1. Aitken LM, Hendrikz JK, Dulhunty JM, Rudd MJ. Hypothermia and associated outcomes in seriously injured trauma patients in a predominantly sub-tropical climate. Resuscitation. 2009;80:217-223. 
2. Beilman GJ, Blondet JJ, Nelson TR, et al. Early hypothermia in severely injured trauma patients is a significant risk factor for multiple organ dysfunction syndrome but not mortality. Ann Surg. 2009;249:845-850.

3. Ireland S, Endacott R, Cameron P, Fitzgerald M, Paul E. The incidence and significance of accidental hypothermia in major trauma-a prospective observational study. Resuscitation. 2011;82:300-306.

4. Martin RS, Kilgo PD, Miller PR, Hoth JJ, Meredith JW, Chang MC. Injury-associated hypothermia: an analysis of the 2004 National Trauma Data Bank. Shock. 2005;24:114-118.

5. Shafi S, Elliott AC, Gentilello L. Is hypothermia simply a marker of shock and injury severity or an independent risk factor for mortality in trauma patients? Analysis of a large national trauma registry. J Trauma. 2005;59:1081-1085.

6. Wang HE, Callaway CW, Peitzman AB, Tisherman SA. Admission hypothermia and outcome after major trauma. Crit Care Med. 2005;33:1296-1301.

7. Arthurs Z, Cuadrado D, Beekley A, et al. The impact of hypothermia on trauma care at the 31 st combat support hospital. Am J Surg. 2006;191:610-614.

8. Helm M, Lampl L, Hauke J, Bock KH. Accidental hypothermia in trauma patients. Is it relevant to preclinical emergency treatment? [in German]. Anaesthesist. 1995;44: $101-107$.

9. Husum H, Olsen T, Murad M, Heng YV, Wisborg T, Gilbert M. Preventing post-injury hypothermia during prolonged prehospital evacuation. Prehosp Disaster Med. 2002;17:23-26.

10. Langhelle A, Lockey D, Harris T, Davies G. Body temperature of trauma patients on admission to hospital: a comparison of anaesthetised and non-anaesthetised patients. Emerg Med J. 2012;29:239-242.

11. Danzl DF. Accidental hypothermia. In: Auerbach PS, ed. Wilderness Medicine. Philadelphia, PA: Mosby Elsevier; 2007:125-159.

12. Durrer B, Brugger H, Syme D, International Commission for Mountain Emergency Medicine. The medical on-site treatment of hypothermia: ICAR-MEDCOM recommendation. High Alt Med Biol. 2003;4:99-103.

13. Søreide K. Clinical and translational aspects of hypothermia in major trauma patients: from pathophysiology to prevention, prognosis and potential preservation. Injury. 2014;45:647-654.

14. Auerbach PS, Donner HJ, Weiss EA, eds. Field Guide to Wilderness Medicine. 3rd ed. St. Louis, MO: Mosby Elsevier; 2008.

15. Giesbrecht GG. Hypothermia, Frostbite, and Other Cold Injuries: Prevention, Survival, Rescue and Treatment. Seattle, WA: Mountaineers Books; 2006.

16. Johnson CF. Oxford Handbook of Expedition and Wilderness Medicine. Oxford: Oxford University Press; 2008.

17. Socialstyrelsen. Hypothermia: Cold Injuries and Cold Water Near Drowning. Stockholm: The National Board of Health and Welfare (Socialstyrelsen); 2002.

18. State of Alaska. State of Alaska Cold Injuries and Cold Water Near Drowning Guidelines (Rev. 1/2005). Transport
Guidelines Severely Hypothermic Patients. Available at: http://www.hypothermia.org/protocol.htm. Accessed January 4, 2012.

19. Brown DJ, Brugger H, Boyd J, Paal P. Accidental hypothermia. N Engl J Med. 2012;367:1930-1938.

20. Brugger H, Durrer B, Elsensohn F, et al. ICAR MEDCOM. Resuscitation of avalanche victims: evidence-based guidelines of the International Commission for Mountain Emergency Medicine (ICAR MEDCOM): intended for physicians and other advanced life support personnel. Resuscitation. 2013;84:539-546.

21. Henriksson O, Lundgren P, Kuklane K, Holmér I, Naredi $\mathrm{P}$, Bjornstig U. Protection against cold in prehospital care: evaporative heat loss reduction by wet clothing removal or the addition of a vapor barrier - a thermal manikin study. Prehosp Disaster Med. 2012;27:53-58.

22. McArdle WD, Katch FL, Katch VL. Exercise Physiology: Energy, Nutrition, and Human Performance. 4th ed. Baltimore, MD: Williams \& Wilkins; 1996.

23. Du Bois D, Du Bois EF. A formula to estimate the approximate surface area if height and weight be known. Arch Intern Med. 1916;17:863-871.

24. Durnin JV, Womersley J. Body fat assessed from total body density and its estimation from skinfold thickness: measurements on 481 men and women aged from 16 to 72 years. Br J Nutr. 1974;32:77-97.

25. ISO 8996:2004. Ergonomics of the thermal environmentdetermination of metabolic rate. Geneva, Switzerland: International Standards Organisation; 2004.

26. Hayward JS, Eckerson JD, Kemna D. Thermal and cardiovascular changes during three methods of resuscitation from mild hypothermia. Resuscitation. 1984;11:21-33.

27. Webb GE. Comparison of esophageal and tympanic temperature monitoring during cardiopulmonary bypass. Anesth Analg. 1973;52:729-733.

28. ISO 9886:2004. Ergonomics-evaluation of thermal strain by physiological measurements. Geneva, Switzerland: International Standards Organisation; 2004.

29. Mekjavić IB, Rempel ME. Determination of esophageal probe insertion length based on standing and sitting height. J Appl Physiol. 1990;69:376-379.

30. Lundgren P, Henriksson O, Kuklane K, Holmér I, Naredi P, Bjornstig U. Validity and reliability of the Cold Discomfort Scale: a subjective judgement scale for the assessment of patient thermal state in a cold environment. J Clin Monit Comput. 2014;28:287-291.

31. Miller RD, Eriksson LI, Fleisher L, Wiener-Kronish JP, Young WL, eds. Miller's Anesthesia. 7th ed. Philadelphia, PA: Churchill Livingstone; 2009.

32. Raven PB, Niki I, Dahms TE, Horvath SM. Compensatory cardiovascular responses during an environmental cold stress, 5 degrees C. J Appl Physiol. 1970;29:417-421.

33. National Association of Emergency Medical Technicians (U.S.). PHTLS: Prehospital Trauma Life Support. 7th ed. St. Louis, MO: Mosby Jems/Elsevier; 2011.

34. Thomassen $\varnothing$, Færevik $H, \varnothing$ sterås $\varnothing$, et al. Comparison of three different prehospital wrapping methods for 
preventing hypothermia-a crossover study in humans. Scand J Trauma Resusc Emerg Med. 2011;19:41.

35. Tsuei BJ, Kearney PA. Hypothermia in the trauma patient. Injury. 2004;35:7-15.

36. Giesbrecht GG. Cold stress, near drowning and accidental hypothermia: a review. Aviat Space Environ Med. 2000; 71:733-752.
37. Henriksson O, Lundgren JP, Kuklane K, Holmér I, Bjornstig U. Protection against cold in prehospital carethermal insulation properties of blankets and rescue bags in different wind conditions. Prehosp Disaster Med. 2009;24:408-415. 